\title{
Chapter 8 \\ On uniform convergence and some related types of convergence
}

\author{
ROBERT DROZDOWSKI, JACEK JĘDRZEJEWSKI, STANISŁAW \\ KOWALCZYK, AGATA SOCHACZEWSKA
}

2010 Mathematics Subject Classification: 40A30, 54E99.

Key words and phrases: pointwise convergence, almost-uniform convergence, quasi-uniform convergence, completely regular spaces, pseudo-compact spaces .

\subsection{Convergence of nets of functions}

There are known several types of convergence of nets of functions. The best known types of convergence in the space of functions are pointwise and uniform convergence. One can find many kinds of convergence which are called in different manner. Some of them are weaker than uniform convergence but stronger than pointwise one, some of them are even stronger than uniform convergence. In the work we will consider mainly convergence of nets of functions defined on a topological space with values in a metric space. We investigate connections between different kinds of convergence and preserving of continuity and integrability of limit functions.

Throughout the article, $(X, \top)$ will denote a topological space and $(Y, \rho)$ will denote a metric space. We start from definitions of some types of convergence of nets of functions $f: X \longrightarrow Y$. The best known types of convergence are pointwise and uniform convergence. 
Definition 8.1. We say that a net $\left\{f_{j}: j \in J\right\}$ of functions defined on a topological space $X$ with values in a metric space $(Y, \rho)$ is pointwise convergent to a function $f: X \longrightarrow Y$ if

$$
\forall_{x \in X} \forall_{\varepsilon>0} \exists_{j_{0} \in J} \forall_{j \geq j_{0}}\left(\rho\left(f_{j}(x), f(x)\right)<\varepsilon\right) .
$$

Definition 8.2. We say that a net $\left\{f_{j}: j \in J\right\}$ of functions defined on a topological space $X$ with values in a metric space $(Y, \rho)$ is uniformly convergent to a function $f: X \longrightarrow Y$ if

$$
\forall_{\varepsilon>0} \exists_{j_{0} \in J} \forall_{j \geq j_{0}} \forall_{x \in X}\left(\rho\left(f_{j}(x), f(x)\right)<\varepsilon\right) .
$$

Relations between these two kinds of convergence are well known, we will use them without any further remarks.

Let us make a review of those kinds of convergence which will be considered. The first one is so called quasi-uniform convergence. It is sometimes called Arzelá convergence after the name of the author, who introduced this kind of convergence into mathematical life.

Definition 8.3. ([1], [4], [12]) A pointwise convergent net $\left\{j_{j}: j \in J\right\}$ of functions defined in a topological space $X$ with values in a metric space $(Y, \rho)$ is called quasi-uniformly convergent to a function $f: X \longrightarrow Y$ if

$$
\forall_{\mathcal{E}>0} \forall_{j \in J} \exists_{k_{j}} \exists_{l_{1}, \ldots, l_{k_{j}} \geq j} \forall_{t \in X}\left(\min \left\{\rho\left(f_{l_{i}}(t), f(t)\right): i \in\left\{1, \ldots, k_{j}\right\}\right\}<\varepsilon\right) .
$$

Of course everybody can see that this kind of convergence is weaker than uniform convergence but stronger than pointwise convergence.

In the literature there was considered another kind of convergence which is also a bit weaker than uniform convergence and stronger than pointwise convergence. It is called almost-uniform convergence.

For any point $x$ in a topological space $X$ by $\mathcal{B}_{x}$ we will denote the class of all open neighbourhoods of the point $x$.

Definition 8.4. ([5], [13]). A pointwise convergent net $\left\{f_{j}: j \in J\right\}$ of functions defined in a topological space $X$ with values in a metric space $(Y, \rho)$ is called almost-uniformly convergent to a function $f: X \longrightarrow Y$ if

$$
\forall_{x \in X} \forall_{\varepsilon>0} \forall_{j \in J} \exists_{j_{x} \geq j} \exists_{U_{x} \in \mathcal{B}_{x}} \forall_{t \in U_{x}}\left(\rho\left(f_{j_{x}}(t), f(t)\right)<\varepsilon\right) .
$$

In further part of the article we will make use of the following denotations. The symbol $\mathcal{C}(X, Y)$ will denote the class of all continuous functions defined in a topological space $X$ with values in a metric space $Y$. Similarly, the symbol 
$\mathcal{F}(X, Y)$ will denote the class of all functions defined in a topological space $X$ with values in a metric space $Y$. Moreover, let $\mathbb{R}^{+}=(0, \infty)$.

Definition 8.5. ([14], [2], [8], [10], [11]). A net $\left\{f_{j}: j \in J\right\}$ of functions defined in a topological space $X$ with values in a metric space $(Y, \rho)$ is said to be convergent to a function $f: X \longrightarrow Y$ in the sense of Whitney (shortly W-convergent) if for each function $\varphi$ from $\mathcal{C}\left(X, \mathbb{R}^{+}\right)$there exists $j_{0} \in J$ such that

$$
\rho\left(f_{j}(x), f(x)\right)<\varphi(x)
$$

for each $x \in X$ and for each $j \in J$ such that $j \geq j_{0}$.

This kind of convergence is even stronger than uniform one, since each constant function is continuous.

Quasi-uniform convergence and convergence in the sense of Whitney can be combined, hence we can define:

Definition 8.6. ([6]) A net $\left\{f_{j}: j \in J\right\}$ of functions defined in a topological space $X$ with values in a metric space $(Y, \rho)$ is said to be convergent to a function $f: X \longrightarrow Y$ in the sense of Arzelá-Whitney (shortly AW-convergent) if this net is pointwise convergent to the function $f$ and for every $\varphi \in \mathcal{C}\left(X, \mathbb{R}^{+}\right)$, every $j_{0}$ in $J$ there exists a finite subset $J_{1}$ of $J$ such that $j \geq j_{0}$ for each $j \in J_{1}$ and

$$
\min \left\{\rho\left(f_{j}(x), f(x)\right): j \in J_{1}\right\}<\varphi(x) \text { if } x \in X .
$$

Similarly, combining Whitney convergence with almost-uniform convergence we are able to define another kind of convergence.

Definition 8.7. A net $\left\{f_{j}: j \in J\right\}$ of functions defined in a topological space with values in a metric space $(Y, \rho)$ is said to be almost-Whitney convergent to a function $f: X \longrightarrow Y$ if this net is pointwise convergent to function $f$ and for every $x \in X, \varphi \in \mathcal{C}\left(X, \mathbb{R}^{+}\right)$, and for every $j$ in $J$ there exist $j_{x} \in J$ and a neighbourhood $U_{x}$ of $x$ such that

$$
\rho\left(f_{j}(t), f(t)\right)<\varphi(x)
$$

for each $t \in U_{x}$.

We have defined 7 kinds of convergence of nets of functions. In further parts of the work we will consider some properties of these types of convergence. 


\subsection{Relations between considered types of convergence}

Since positive constant function $\varphi_{\varepsilon}: X \longrightarrow \mathbb{R}^{+}, \varphi_{\varepsilon}(x)=\varepsilon$ for all $x$ in $X$ belongs to $\mathcal{C}\left(X, \mathbb{R}^{+}\right)$, it is easy to observe.

Remark 8.8. If a net $\left\{f_{j}: j \in J\right\}$ of functions defined in a topological space $X$ with values in a metric space $(Y, \rho)$ is W-convergent to $f: X \longrightarrow Y$ then it is uniformly convergent to $f$.

Remark 8.9. If a net $\left\{f_{j}: j \in J\right\}$ of functions defined in a topological space $X$ with values in a metric space $(Y, \rho)$ is almost-Whitney convergent to $f$ : $X \longrightarrow Y$ then it is almost-uniformly convergent to $f$.

Remark 8.10. If a net $\left\{f_{j}: j \in J\right\}$ of functions defined in a topological space $X$ with values in a metric space $(Y, \rho)$ is AW-convergent to $f: X \longrightarrow Y$ then it is quasi-uniformly convergent to $f$.

Immediately from definitions of quasi-uniform convergence and almostuniform convergence, we infer that if a net $\left\{f_{j}: j \in J\right\}$ of functions from $\mathcal{F}(X, Y)$ is quasi-uniformly convergent or almost-uniformly convergent to $f: X \longrightarrow Y$ then this net is pointwise convergent to $f$.

Moreover, it is obvious that each uniformly convergent net of functions $\left\{f_{j}: j \in J\right\}$ from $\mathcal{F}(X, Y)$ is quasi-uniformly convergent, almost-uniformly convergent and pointwise convergent.

Similarly, it is easy to see that each W-convergent net of functions from $\mathcal{F}(X, Y)$ is AW-convergent and almost-Whitney convergent.

Theorem 8.11. Let $(X, \top)$ be a topological space and let $(Y, \rho)$ be a metric space. If a net $\left\{f_{j}: j \in J\right\}$ of functions from $\mathcal{F}(X, Y)$ is almost-uniformly convergent to $f: X \longrightarrow Y$ then it is almost-Whitney convergent to $f$.

Proof. Let a net $\left\{f_{j}: j \in J\right\}$ be almost-uniformly convergent to a function $f: X \longrightarrow Y$. Fix $x \in X, j \in J$ and $\varphi \in \mathcal{C}\left(X, \mathbb{R}^{+}\right)$. Then $\varepsilon=\frac{1}{2} \varphi(x)>0$. By continuity of $\varphi$, there exists a neighbourhood $U_{1}$ of $x$ such that $\varphi(t)>\varepsilon$ for all $t \in U_{1}$. Since $\left\{f_{j}: j \in J\right\}$ is almost-uniformly convergent to $f$, there exist $j_{x} \geq j$ and a neighbourhood $U_{2}$ of $x$ such that $\rho\left(f_{j_{x}}(t), f(t)\right)<\varepsilon$ for all $t \in U_{2}$.

Let $U=U_{1} \cap U_{2}$. Then $U$ is a neighbourhood of $x$ and

$$
\rho\left(f_{j_{x}}(t), f(t)\right)<\varepsilon<\varphi(t) \quad \text { if } \quad t \in U .
$$

Since $\left\{f_{j}: j \in J\right\}$ is pointwise convergent to $f$, it proves that $\left\{f_{j}: j \in J\right\}$ is almost-Whitney convergent to $f$. 
Corollary 8.12. Let $(X, \top)$ be a topological space and let $(Y, \rho)$ be a metric space. If a net $\left\{f_{j}: j \in J\right\}$ of functions from $\mathcal{F}(X, Y)$ is uniformly convergent to $f: X \longrightarrow Y$ then it is almost-Whitney convergent to $f$.

Corollary 8.13. For every topological space $(X, \mathcal{T})$ and every metric space $(Y, \rho)$ almost-uniform convergence and almost Whitney convergence are equivalent in $\mathcal{F}(X, Y)$.

Remark 8.14. If a net $\left\{f_{j}: j \in J\right\}$ of functions from $\mathcal{F}(X, Y)$ is pointwise convergent to $f: X \rightarrow Y$ and it has a subnet which is $\mathrm{W}$-convergent then it is AW-convergent to $f$.

The diagram shows the relations among the considered kinds of convergence.

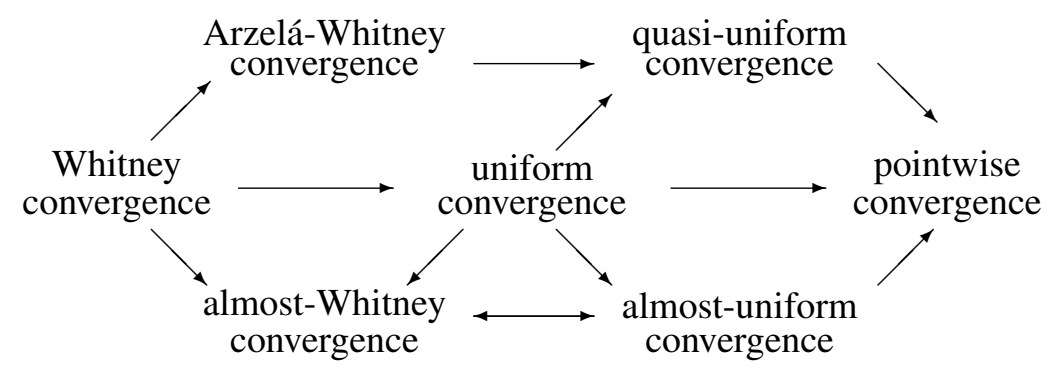

No other implication can be stated as a few examples show.

Example 8.15. Pointwise convergence implies neither almost-uniform convergence nor quasi-uniform convergence.

Let $X=[0,1]$ and $Y=\mathbb{R}$ and $f_{n}(x)=x^{n} \quad$ if $\quad x \in[0,1]$. Then the sequence $\left(f_{n}\right)_{n=1}^{\infty}$ is pointwise convergent to the function

$$
f(x)= \begin{cases}0, & \text { if } x \in[0,1) \\ 1, & \text { if } x=1\end{cases}
$$

but this sequence is not almost-uniformly convergent to $f$. Similarly, it is not quasi-uniformly convergent to $f$.

Example 8.16. Almost-uniform convergence does not imply quasi-uniform convergence.

Let $X=[0,1)$ and $Y=\mathbb{R}$ and $f_{n}(x)=x^{n}$ if $x \in[0,1)$. Then the sequence $\left(f_{n}\right)_{n=1}^{\infty}$ is almost-uniformly convergent to the zero function, but this sequence is not quasi-uniformly convergent to $f$. 
Example 8.17. Neither AW-convergence nor quasi-uniform convergence imply almost uniform convergence.

Let $X=[-1,1]$ and $Y=\mathbb{R}$ and if $n$ is even positive integer then

$$
f_{n}(x)= \begin{cases}0, & \text { if } \quad x \in[-1,0] \cup\left[\frac{1}{n}, 1\right] \\ 1, & \text { if } \quad x \in\left(0, \frac{1}{n}\right)\end{cases}
$$

and if $n$ is odd positive integer then

$$
f_{n}(x)=\left\{\begin{array}{lll}
0, & \text { if } & x \in\left[-1,-\frac{1}{n}\right] \cup[0,1] \\
1, & \text { if } & x \in\left(-\frac{1}{n}, 0\right)
\end{array}\right.
$$

Then the sequence $\left(f_{n}\right)_{n=1}^{\infty}$ is AW-convergent (and also quasi-uniformly convergent) to the zero function in $[-1,1]$, but this sequence is not almostuniformly convergent to $f$.

Further examples state that neither quasi-uniform convergence nor almostuniform convergence imply uniform convergence.

Example 8.18. None of the uniform convergence, almost-Whitney convergence, quasi-uniform convergence implies AW-convergence.

Let $f_{n}:(0,1) \longrightarrow[0,1]$ be defined as follows:

$$
f_{n}(x)=\frac{1}{n} \quad \text { if } x \in(0,1) .
$$

Then the sequence $\left(f_{n}\right)_{n=1}^{\infty}$ is not AW-convergent to zero function in $(0,1)$, but it is uniformly convergent, almost Whitney and quasi-uniformly convergent.

Example 8.19. Almost Whitney convergence implies neither uniform convergence nor AW-convergence.

Let $f_{n}(x)=x^{n}$ and $f(x)=0, x \in(0,1)$. Then $\left\{f_{n}: n \in \mathbb{N}\right\}$ is almost Whitney convergent to $f$ but it is neither uniformly convergent nor AW-convergent. Hence it is not W-convergent.

\subsection{Continuity of limit of nets of continuous functions}

Everyone does know that if a net of continuous functions is uniformly convergent to a function $f$, then $f$ is continuous as well and that pointwise convergence is too weak to get the continuity of the limit function.

The following facts concerning quasi-uniform convergence are well known. 
Theorem 8.20. ([13]). If a net of continuous functions defined in a topological space $X$ with values in a metric space $(Y, \rho)$ is quasi-uniformly convergent to a function $f: X \longrightarrow Y$ then $f$ itself is continuous.

Proof. Let $\left\{f_{j}: j \in J\right\}$ be any net of functions from $\mathcal{C}(X, Y)$.

Let $\varepsilon$ be a fixed positive number and $x_{0}$ be a fixed point from $X$. There exists an index $j_{0} \in J$ such that

$$
\rho\left(f_{j}\left(x_{0}\right), f\left(x_{0}\right)\right)<\frac{\varepsilon}{3}
$$

for all $j$ not less than $j_{0}$.

There is a finite subset $J_{1}$ of $J$ such that if $j_{p} \in J_{1}$ then $j_{p} \geq j_{0}$ and for each $x$ from $X$,

$$
\min \left\{\rho\left(f_{j}(x), f(x)\right): j \in J_{1}\right\}<\frac{\varepsilon}{3} .
$$

Each of the functions $f_{j}$ is continuous so for each $j$ from $J_{1}$ there is a neighbourhood $U_{j}$ of the point $x_{0}$ such that

$$
\rho\left(f_{j}(x), f_{j}\left(x_{0}\right)\right)<\frac{\varepsilon}{3}, \quad \text { if } \quad x \in U_{j} .
$$

Let $U=\bigcap_{j \in J_{1}} U_{j}$. Fix any point $u$ from the set $U$. For this element there is $j_{u}$ such that

$$
\rho\left(f_{j_{u}}(u), f(u)\right)<\frac{\varepsilon}{3} .
$$

Then

$$
\begin{gathered}
\rho\left(f(u), f\left(x_{0}\right)\right) \leq \\
\leq \rho\left(f(u), f_{j_{u}}(u)\right)+\rho\left(f_{j_{u}}(u), f_{j_{u}}\left(x_{0}\right)\right)+\rho\left(f_{j_{u}}\left(x_{0}\right), f\left(x_{0}\right)\right)<\varepsilon .
\end{gathered}
$$

In that way we have proved that the limit function $f$ is continuous at $x_{0}$, hence it is continuous in $X$.

Theorem 8.21. Let $X$ be a compact topological space and let $\left\{f_{j}: j \in J\right\}$ be a pointwise convergent net of continuous functions defined in $X$ with values in a metric space $(Y, \rho)$. If the limit function $f: X \longrightarrow Y$ is continuous as well, then the net $\left\{f_{j}: j \in J\right\}$ is quasi-uniformly convergent to $f$.

Proof. It is clear that the first condition of quasi-uniform convergence is fulfilled.

Let $\varepsilon$ be any fixed positive number and $j_{0}$ any index from $J$.

Pointwise convergence of the net $\left\{f_{j}: j \in J\right\}$ to the function $f$ implies that for each $x \in X$ there exists an index $j_{x}$ such that $j_{x} \geq j_{0}$ and

$$
\rho\left(f_{j}(x), f(x)\right)<\frac{\varepsilon}{3}, \text { if } j \geq j_{x} \geq j_{0} .
$$


Functions $f_{j_{x}}$ and $f$ are continuous, so there is a neighborhood $U_{x}$ of the point $x$ such that

$$
\rho\left(f_{j_{x}}(u), f_{j_{x}}(x)\right)<\frac{\varepsilon}{3} \quad \text { and } \quad \rho(f(u), f(x))<\frac{\varepsilon}{3},
$$

if $u \in U_{x}$. Then

$$
\rho\left(f_{j_{x}}(u), f(u)\right)<\varepsilon,
$$

for each $u \in U_{x}$.

The class of sets $\left\{U_{x}: x \in X\right\}$ consists of open sets and forms, of course, a cover of the compact space $X$. Then there exists a finite subset $\left\{x_{1}, \ldots, x_{k}\right\}$ of the set $X$ such that

$$
X=\bigcup_{i=1}^{k} U_{x_{i}} .
$$

Let $v$ be any point from the set $X$, then there is a $p$ from the set $\{1, \ldots, k\}$ such that $v \in U_{x_{p}}$. Thus condition (8.1) from Definition 8.3. is fulfilled.

Example 8.22. Compactness of $X$ can not be omitted in the previous theorem.

Let $f_{n}:[0, \infty) \longrightarrow \mathbb{R}$ be defined as follows:

$$
f_{n}(x)=\left\{\begin{aligned}
0, & \text { if } x \in[0, n], \\
x-n, & \text { if } x \in(n, n+1], \\
1, & \text { if } x \in(n+1, \infty) .
\end{aligned}\right.
$$

It is easy to see that all functions $f_{n}$ are continuous, the sequence $\left(f_{n}\right)_{n=1}^{\infty}$ is pointwise convergent to the zero function in $[0, \infty)$ but this sequence is not quasi-uniformly convergent.

Theorem 8.23. If a net of continuous functions defined in a topological space $X$ with values in a metric space $(Y, \rho)$ is almost-uniformly convergent to a function $f: X \longrightarrow Y$ then $f$ itself is continuous.

Proof. Let $\left\{f_{j}: j \in J\right\}$ be any almost-uniformly convergent net of continuous functions defined in $X$ with values in $(Y, \rho)$.

Let $\varepsilon$ be an arbitrary positive number and $x_{0}$ be a fixed point in $X$. Since the net $\left\{f_{j}: j \in J\right\}$ is pointwise convergent to a function $f: X \longrightarrow Y$, there exists an index $j_{0} \in J$ such that for each $j \geq j_{0}$

$$
\rho\left(f_{j}\left(x_{0}\right), f\left(x_{0}\right)\right)<\frac{\varepsilon}{3} .
$$

Since the considered net is almost-uniformly convergent to $f$, there are $j_{x_{0}} \geq j_{0}$ and a neighbourhood $U_{1}$ of the point $x_{0}$ such that 


$$
\rho\left(f_{j_{x_{0}}}(t), f(t)\right)<\frac{\varepsilon}{3}
$$

for each $t \in U_{1}$.

Function $f_{j_{x_{0}}}$ is continuous, then there exists a neighbourhood $U_{2}$ of the point $x_{0}$ such that

$$
\rho\left(f_{j_{x_{0}}}(t), f_{j_{x_{0}}}\left(x_{0}\right)\right)<\frac{\varepsilon}{3} .
$$

Then for each $x \in U_{1} \cap U_{2}$ we have:

$$
\begin{gathered}
\rho\left(f(x), f\left(x_{0}\right)\right) \leq \\
\leq \rho\left(f(x), f_{j_{x_{0}}}(x)\right)+\rho\left(f_{j_{x_{0}}}(x), f_{j_{x_{0}}}\left(x_{0}\right)\right)+\rho\left(f_{j_{x_{0}}}\left(x_{0}\right), f\left(x_{0}\right)\right)<\varepsilon,
\end{gathered}
$$

which proves that $f$ is continuous at the point $x_{0}$.

Hence this function is continuous in $X$.

Theorem 8.24. Let $X$ be arbitrary topological space and let $\left\{f_{j}: j \in J\right\}$ be a pointwise convergent net of continuous functions defined in $X$ with values in a metric space $(Y, \rho)$. If the limit function $f: X \longrightarrow \mathbb{R}$ is continuous as well, then the net $\left\{f_{j}: j \in J\right\}$ is almost-uniformly convergent to $f$.

Proof. Let us fix any point $x_{0}$ from the space $X$.Take any $\varepsilon>0$. Then there exists $j_{0} \in J$ such that

$$
\rho\left(f_{j}\left(x_{0}\right), f\left(x_{0}\right)\right)<\frac{\varepsilon}{3} .
$$

The function $f_{j_{0}}$ is continuous, then there exists a neighbourhood $U_{1}$ of the point $x_{0}$ such that

$$
\rho\left(f_{j_{0}}(x), f_{j_{0}}\left(x_{0}\right)\right)<\frac{\varepsilon}{3}
$$

for each $x \in U_{1}$.

The function $f$ is continuous as well, then there exists a neighbourhood $U_{2}$ of the point $x_{0}$ such that

$$
\rho\left(f(x), f\left(x_{0}\right)\right)<\frac{\varepsilon}{3}
$$

for each $x \in U_{2}$.

Hence, if $x \in U_{1} \cap U_{2}$ then

$$
\begin{gathered}
\rho\left(f_{j_{0}}(x), f(x)\right) \leq \\
\leq \rho\left(f_{j_{0}}(x), f_{j_{0}}\left(x_{0}\right)\right)+\rho\left(f_{j_{0}}\left(x_{0}\right), f\left(x_{0}\right)\right)+\rho\left(f\left(x_{0}\right), f(x)\right)<\varepsilon .
\end{gathered}
$$

Thus we have proved that condition (8.2) is fulfilled. Hence the net $\left\{f_{j}: j \in J\right\}$ is almost uniformly convergent to $f$. 
Remark 8.25. Applying relations among the considered kinds of convergence shown in section 2 and Theorems 8.20 and 8.23, we can easily see that if a net $\left\{f_{j}: j \in J\right\}, f_{j}: X \rightarrow Y$ of continuous functions is AW-convergent or almost Whitney convergent or Whitney convergent to a function $f: X \rightarrow Y$ then $f$ is continuous too.

Remark 8.26. Applying properties of limits of nets of continuous functions considered in section 3 it is easy to see that in $\mathcal{C}(X, Y)$ quasi-uniform convergence implies almost-uniform convergence, and if $X$ is compact then these kinds of convergence are equivalent.

Let $(X, \top)$ be a topological space and $\mathcal{J}$ be a $\sigma$-ideal of subsets of this space, which contains no non-degenerate interval and $(Y, \rho)$ be a metric space. We say that a function $f: X \longrightarrow Y$ has property $\mathcal{P} \mathcal{J}$-almost everywhere if the set of points at which function $f$ does not have this property belongs to $\mathcal{J}$. So, a function $f$ is continuous $\mathcal{J}$-almost everywhere in $X$ if the set $\mathcal{D}_{f}$ of points of discontinuity of the function $f$ belongs to $\mathcal{J}$.

Previous theorems can be generalized a little in the case of sequences of functions. We can get the following:

Theorem 8.27. ([7]) Let $\left(f_{n}\right)_{n=1}^{\infty}$ be a sequence of functions defined in a topological space $X$ with values in a metric space $(Y, \rho)$. If each of the functions $f_{n}$ is continuous $\mathcal{J}$-almost everywhere in $X$ and the sequence $\left(f_{n}\right)_{n=1}^{\infty}$ is quasiuniformly convergent to a function $f: X \longrightarrow Y$, then $f$ itself is also continuous J-almost everywhere in $X$.

Proof. Let $E_{n}$ denote the set of points of continuity of the function $f_{n}$. Now let $E=\bigcap_{n=1}^{\infty} E_{n}$. It is evident that $X \backslash E \in \mathcal{J}$, since $X \backslash E_{n} \in \mathcal{J}$ and $\mathcal{J}$ is $\sigma$-ideal.

Let $x_{0} \in E$. For arbitrary positive $\varepsilon$ there exists a positive integer $n_{0}$ such that

$$
\rho\left(f_{n}\left(x_{0}\right), f\left(x_{0}\right)\right)<\frac{\varepsilon}{3} \quad \text { if } \quad n \geq n_{0} .
$$

In view of condition (8.1) we can conclude that there exist $n_{1}, \ldots, n_{k}$ such that $n_{1} \geq n, \ldots, n_{k} \geq n_{0}$ and

$$
\rho\left(f_{n_{1}}(t), f(t)\right)<\frac{\varepsilon}{3} \vee \ldots \vee \rho\left(f_{n_{k}}(t), f(t)\right)<\frac{\varepsilon}{3}
$$

for all $t \in X$.

Each of the functions $f_{n_{i}}$ is continuous at $x_{0}$, then there exists a neighborhood $U_{0}$ of $x_{0}$ such that

$$
\rho\left(f_{n_{i}}(t), f_{n_{i}}\left(x_{0}\right)\right)<\frac{\varepsilon}{3} .
$$


for each $t \in U_{0}$ and $i \in\{1, \ldots, k\}$.

Let $x \in U_{0}$ and let $n_{i_{0}} \in\left\{n_{1}, \ldots, n_{k}\right\}$ be such that

$$
\rho\left(f_{n_{i_{0}}}(x), f(x)\right)<\frac{\varepsilon}{3} .
$$

Thus

$$
\begin{gathered}
\rho\left(f(x), f\left(x_{0}\right)\right) \leq \\
\leq \rho\left(f(x), f_{n_{i_{0}}}(x)\right)+\rho\left(f_{n_{i_{0}}}(x), f_{n_{i_{0}}}\left(x_{0}\right)\right)+\rho\left(f_{n_{i_{0}}}\left(x_{0}\right), f\left(x_{0}\right)\right)<\varepsilon,
\end{gathered}
$$

which proves continuity of $f$ at the point $x_{0}$ from the set $E$.

Since $X \backslash E \in \mathcal{J}$, the function $f$ is continuous $\mathcal{J}$-almost everywhere.

Theorem 8.28. Let $\left(f_{n}\right)_{n=1}^{\infty}$ be a sequence of functions defined in a topological space $X$ with values in a metric space $(Y, \rho)$. If each of the functions $f_{n}$ is continuous J-almost everywhere in $X$ and the sequence $\left(f_{n}\right)_{n=1}^{\infty}$ is almostuniformly convergent to a function $f: X \longrightarrow Y$, then $f$ is also continuous J-almost everywhere in $X$.

Proof. Let $E_{n}$ denote the set of points of continuity of the function $f_{n}$. Moreover, let

$$
E=\bigcap_{n=1}^{\infty} E_{n} .
$$

Since $X \backslash E_{n} \in \mathcal{J}$ for each positive integer $n$, also $X \backslash E \in \mathcal{J}$.

Let $x_{0} \in E$. For arbitrary positive $\varepsilon$ there exists a positive integer $n_{0}$ such that

$$
\rho\left(f_{n}\left(x_{0}\right), f\left(x_{0}\right)\right)<\frac{\varepsilon}{3} \quad \text { if } \quad n \geq n_{0} .
$$

In view of condition (8.2) we infer that there exist $n_{1}$ and a neighbourhood $U_{1}$ of $x_{0}$ such that $n_{1} \geq n_{0}$ and

$$
\rho\left(f_{n_{1}}(t), f(t)\right)<\frac{\varepsilon}{3} \quad \text { if } \quad t \in U_{1} .
$$

The function $f_{n_{1}}$ is continuous at $x_{0}$, then there exists a neighborhood $U_{2}$ of $x_{0}$ such that

$$
\rho\left(f_{n_{1}}(t), f_{n_{1}}\left(x_{0}\right)\right)<\frac{\varepsilon}{3} \quad \text { if } \quad t \in U_{2} .
$$

If now $x \in U_{1} \cap U_{2}$, then

$$
\begin{gathered}
\rho\left(f(x), f\left(x_{0}\right)\right) \leq \\
\leq \rho\left(f(x), f_{n_{1}}(x)\right)+\rho\left(f_{n_{1}}(x), f_{n_{1}}\left(x_{0}\right)\right)+\rho\left(f_{n_{1}}\left(x_{0}\right), f\left(x_{0}\right)\right)<\varepsilon,
\end{gathered}
$$

which proves that $f$ is continuous at the point $x_{0}$ from the set $E$. 
Since $X \backslash E \in \mathcal{J}$, the function $f$ is continuous $\mathcal{J}$-almost everywhere.

\subsection{Integrability of limit of sequences of Riemann integrable functions}

It is evident that a real function defined in a compact interval is Riemann integrable if and only if the set of discontinuity points of such function has Lebesgue measure zero. The class of sets with Lebesgue measure zero forms a $\sigma$-ideal of sets. Hence one can get the following theorems as corollaries from Theorems 8.27 and 8.28.

Theorem 8.29. Let $\left(f_{n}\right)_{n=1}^{\infty}$ be a sequence of Riemann integrable functions in $[0,1]$. If $f:[0,1] \longrightarrow \mathbb{R}$ is a quasi-uniform limit of the sequence $\left(f_{n}\right)_{n=1}^{\infty}$, then $f$ is also Riemann integrable function.

Theorem 8.30. Let $\left(f_{n}\right)_{n=1}^{\infty}$ be a sequence of Riemann integrable functions in $[0,1]$. If $f:[0,1] \longrightarrow \mathbb{R}$ is an almost-uniform limit of the sequence $\left(f_{n}\right)_{n=1}^{\infty}$, then $f$ is also Riemann integrable function.

Integrability of such limits is not sufficient to prove that the Riemann integral of it is equal to the limit of sequence of integrals of functions $f_{n}$.

Example 8.31. Let $\left(f_{n}\right)_{n=1}^{\infty}$ be defined as follows:

$$
f_{n}(x)=\left\{\begin{aligned}
n^{2} x, & \text { if } x \in\left[0, \frac{1}{n}\right], \\
2 n^{2}-n^{2} x, & \text { if } x \in\left(\frac{1}{n}, \frac{2}{n}\right), \\
0, & \text { if } x \in\left[\frac{2}{n}, 2\right] .
\end{aligned}\right.
$$

Moreover let $f$ be zero function in the interval $[0,2]$. Then the sequence $\left(f_{n}\right)_{n=1}^{\infty}$ is quasi-uniformly and almost-uniformly convergent to $f$. But

$$
\lim _{n \rightarrow \infty} \int_{0}^{2} f_{n}(x) d x=1 \neq 0=\int_{0}^{2} \lim _{n \longrightarrow \infty} f_{n}(x) d x .
$$

However, if we assume that almost-uniformly (or quasi-uniformly) convergent sequence $\left(f_{n}\right)_{n=1}^{\infty}$ of Riemann integrable functions is majorized by a Riemann (or Lebesgue) integrable function, then its limit is not only Riemann integrable, but its integral is a limit of integrals of $f_{n}$. Before we prove those theorems we will need some theorems concerning to Lebesgue integral. It is quite known that if a real function is Riemann integrable on a compact interval 
$[a, b]$, then it is also Lebesgue integrable on $[a, b]$ and those two integrals are equal.

To make a distinction between Riemann integral and Lebesgue integral we will use the denotation $\int_{a}^{b} f(x) d x$ for Riemann integral and $\int_{[a, b]} f d \mu$ for Lebesgue integral of the function $f$ on interval $[a, b]$.

Theorem 8.32. Let $f_{n}:[a, b] \longrightarrow \mathbb{R}$ be Riemann integrable function for each positive integer $n$. If $\lim _{n \rightarrow \infty} f_{n}=f$ and this convergence is quasi-uniform and there exists a Riemann (or Lebesgue) integrable function $g:[a, b] \longrightarrow \mathbb{R}$ such that

$$
\left|f_{n}\right| \leq g,
$$

for $n \geq 1$, then $f$ is Riemann integrable and

$$
\lim _{n \rightarrow \infty} \int_{a}^{b} f_{n}(x) d x=\int_{a}^{b} \lim _{n \longrightarrow \infty} f_{n}(x) d x .
$$

Proof. Riemann integrability of $f$ is implied from Theorem 8.29. From Lebesgue Theorem on dominated convergence one can conclude that $f$ is Lebesgue integrable function and

$$
\begin{aligned}
& \lim _{n \rightarrow \infty} \int_{a}^{b} f_{n}(x) d x=\lim _{n \rightarrow \infty} \int_{[a, b]} f_{n} d \mu= \\
& =\int_{[a, b]} \lim _{n \rightarrow \infty} f_{n} d \mu=\int_{a}^{b} \lim _{n \rightarrow \infty} f_{n}(x) d x .
\end{aligned}
$$

The same arguments can be applied to prove the next theorem.

Theorem 8.33. Let $f_{n}:[a, b] \longrightarrow \mathbb{R}$ be Riemann integrable functions for each positive integer $n$. If $\lim _{n \rightarrow \infty} f_{n}=f$ and this convergence is almost-uniform and there exists a Riemann integrable function $g:[a, b] \longrightarrow \mathbb{R}$ such that

$$
\left|f_{n}\right| \leq g
$$

for $n \geq 1$, then $f$ is Riemann integrable and

$$
\lim _{n \longrightarrow \infty} \int_{0}^{1} f_{n}(x) d x=\int_{0}^{1} \lim _{n \rightarrow \infty} f_{n}(x) d x .
$$




\subsection{Conditions for equivalence of some types of convergence of nets of functions}

Theorem 8.34. Let $(X, \mathcal{T})$ be a completely regular topological space. The following conditions are equivalent:

1. $X$ is pseudo-compact,

2. for every metric space $(Y, \rho) A W$-convergence and quasi-uniform convergence of nets of functions are equivalent in $\mathcal{F}(X, Y)$,

3. AW-convergence and quasi-uniform convergence of nets of functions are equivalent in $\mathcal{F}(X,[0,1])$,

4. for every metric space $(Y, \rho) A W$-convergence and quasi-uniform convergence of nets of functions are equivalent in $\mathcal{C}(X, Y)$,

5. AW-convergence and quasi-uniform convergence of nets of functions are equivalent in $\mathcal{C}(X, Y)$.

Proof. Assume that $X$ is pseudo-compact. Let $\varphi$ be arbitrary function from $\mathcal{C}\left(X, \mathbb{R}^{+}\right)$. Then $\frac{1}{\varphi}$ belongs to $\mathcal{C}(X, \mathbb{R})$. Hence $\frac{1}{\varphi}$ is bounded. Therefore there exists a positive $\delta$ such that $\varphi(x)>\delta$ for all $x \in X$. It follows that AW-convergence and quasi-uniform convergence of nets of functions are equivalent in all considered classes of functions.

Suppose that $X$ is not pseudo-compact. Since $X$ is completely regular, there exists continuous unbounded function $g: X \longrightarrow \mathbb{R}$. Then the function $\varphi: X \longrightarrow \mathbb{R}^{+}$such that $\varphi=\frac{1}{|g|+1}$ belongs to $\mathcal{C}\left(X, \mathbb{R}^{+}\right)$and there exists a sequence $\left(x_{n}\right)_{n=1}^{\infty}$ of points of $X$ such that $\lim _{n \rightarrow \infty} \varphi\left(x_{n}\right)=0$ and $\varphi\left(x_{n}\right)<1$ for each $n \in \mathbb{N}$. Define a sequence of functions $\left(f_{n}\right)_{n=1}^{\infty}$, letting $f_{n}(x)=\varphi\left(x_{n}\right)$ for all $x \in X$.

Then $f_{n} \in \mathcal{C}(X,[0,1])$ and $\left(f_{n}\right)_{n=1}^{\infty}$ is quasi-uniformly convergent to zero function $f$, (in fact $\left(f_{n}\right)_{n=1}^{\infty}$ is uniformly convergent to $f$ ). On the other hand $\left|f_{n}\left(x_{n}\right)-f\left(x_{n}\right)\right|=\varphi\left(x_{n}\right)$ for all $n$ and therefore $\left(f_{n}\right)_{n=1}^{\infty}$ is not AW-convergent to $f$. Hence AW-convergence and quasi-uniform convergence of nets of functions are not equivalent in any considered class of functions.

Theorem 8.35. Let $X$ be almost compact space. If a net $\left\{f_{j}: j \in J\right\}$ of continuous functions $f_{j}: X \longrightarrow Y$ is pointwise convergent to a continuous function $f: X \longrightarrow Y$, then this net is $A W$-convergent to the function $f$.

Proof. Let us fix $j_{0} \in J$ and $\varphi \in \mathcal{C}\left(X, \mathbb{R}^{+}\right)$. For each point $p \in X$ we can choose its neighbourhood $U_{p}$ such that

$$
\frac{3}{4} \cdot \varphi(p)<\varphi(x) \quad \text { if } \quad x \in U_{p} .
$$


We put

$$
W_{p}=B\left(f(p), \frac{1}{8} \cdot \varphi(p)\right)
$$

Thus

$$
\left\{U_{p} \cap f^{-1}\left(W_{p}\right) \cap f_{j}^{-1}\left(W_{p}\right): p \in X \wedge j \geq j_{0}\right\}
$$

is an open cover of $X$. By assumptions, we can select a finite subclass

$$
\left\{U_{p_{k}} \cap f^{-1}\left(W_{p_{k}}\right) \cap f_{j_{k}}^{-1}\left(W_{p_{k}}\right): k \in\{1, \ldots, n\}\right\}
$$

such that

$$
\overline{\bigcup_{k=1}^{n}\left(U_{p_{k}} \cap f^{-1}\left(W_{p_{k}}\right) \cap f_{j_{k}}^{-1}\left(W_{p_{k}}\right)\right)}=X .
$$

Let $x$ be in $X$. Then

$$
x \in \overline{U_{p_{k}} \cap f^{-1}\left(W_{p_{k}}\right) \cap f_{j_{k}}^{-1}\left(W_{p_{k}}\right)}
$$

for some $k$ in $\{1, \ldots, n\}$.

Hence

$$
\varphi(x) \in \varphi\left(\overline{U_{p_{k}}}\right) \subset \overline{\varphi\left(U_{p_{k}}\right)} \subset\left[\frac{3}{4} \cdot \varphi\left(p_{k}\right), \infty\right),
$$

which means that

$$
\frac{3}{4} \cdot \varphi\left(p_{k}\right) \leq \varphi(x)
$$

Furthermore,

$$
f(x) \in \overline{W_{p_{k}}}=\overline{B\left(f\left(p_{k}\right), \frac{1}{8} \cdot \varphi\left(p_{k}\right)\right)} \subset B\left(f\left(p_{k}\right), \frac{1}{4} \cdot \varphi\left(p_{k}\right)\right) .
$$

Analogously,

$$
f_{j_{k}}(x) \in B\left(f\left(p_{k}\right), \frac{1}{4} \cdot \varphi\left(p_{k}\right)\right) .
$$

Thus we infer that

$$
\rho\left(f(x), f_{j_{k}}(x)\right)<\frac{1}{2} \cdot \varphi\left(p_{k}\right)<\varphi(x) .
$$

Finally, letting $J_{1}=\left\{j_{1}, \ldots, j_{n}\right\}$ we can conclude that the net $\left\{f_{j}: j \in J\right\}$ is AW-convergent.

Theorem 8.36. If $X$ is a paracompact Hausdorff space, then the following conditions are equivalent:

(1) $X$ is a compact space,

(2) for each metric space $(Y, \rho)$ the AW-convergence and pointwise convergence coincide in the class $\mathcal{C}(X, Y)$, 
(3) the AW-convergence and pointwise convergence coincide in the class $\mathcal{C}(X,[0,1])$.

Proof. The implication (1) $\Longrightarrow(2)$ is a consequence of Theorem 8.35.

The implication $(2) \Longrightarrow(3)$ is evident.

To prove the implication $(3) \Longrightarrow(1)$, suppose that the space $X$ is not compact. There exists an open cover $\left\{U_{s}: s \in S\right\}$, which has no finite subcover. Since $X$ is paracompact Hausdorff space, there exists a locally finite closed cover $\mathfrak{V}$ consisting of the sets $M_{s}$, where $s \in S$, for which $M_{s} \subset U_{s}$ (see [6], Lemma 5.1.6).

Let $\leq$ be well order in the set $S$ and $\alpha$ be an order type of $(S, \leq)$. Thus the cover $\mathfrak{V}$ can be taken as a transfinite sequence

$$
M_{s_{0}}, \ldots, M_{s_{\xi}}, \ldots, \quad \xi<\alpha .
$$

Now let

$$
D_{\xi}=\bigcup_{\beta \leq \xi} M_{s_{\beta}}, \quad E_{\xi}=X \backslash \bigcup_{\beta \leq \xi} U_{s_{\beta}}
$$

if $\xi<\alpha$.

Then $\mathfrak{D}$, where

$$
\mathfrak{D}=\left\{D_{\xi}: \xi<\alpha\right\},
$$

is a cover of $X$. The sets $D_{\xi}$ and $E_{\xi}$ are disjoint for each $\xi<\alpha$ and closed, since every $D_{\xi}$ is a union of a locally finite class of closed sets and every $E_{\xi}$ is a complement of an open set.

Moreover, if $\beta<\xi$, then

$$
D_{\beta} \subset D_{\xi} \quad \text { and } \quad E_{\xi} \subset E_{\beta} .
$$

The space $X$ is normal, then for each $\xi$ less than $\alpha$ there exists a continuous function $f_{\xi}: X \longrightarrow[0,1]$ such that

$$
f_{\xi}\left(D_{\xi}\right)=\{1\} \quad \text { and } \quad f_{\xi}\left(E_{\xi}\right)=\{0\} .
$$

It is easy to see that the net $\left\{f_{\xi}: \xi<\alpha\right\}$ is pointwise convergent to the function $f$ defined by $f(x)=1$ if $x \in X$.

Let us take a finite sequence

$$
\left\{f_{\xi_{1}}, f_{\xi_{2}}, \ldots, f_{\xi_{n}}\right\}
$$

where

$$
\xi_{1} \leq \xi_{2} \leq \cdots \leq \xi_{n}<\alpha
$$


and a continuous function $\varphi$ given by $\varphi(x)=\frac{1}{2}, x \in X$.

Since $E_{\xi_{n}} \subset E_{\xi_{k}}$ if $k \in\{1, \ldots, n\}$, we have

$$
f_{\xi_{k}}(x)=0 \text { if } x \in E_{\xi_{n}} \text { and } k \leq n .
$$

From this we infer that

$$
\min \left\{\left|f_{\xi_{k}}(x)-f(x)\right|: k \leq n\right\}>\varphi(x) \quad \text { if } \quad x \in E_{\xi_{n}} .
$$

In that way we have proved that the net $\left\{f_{\xi}: \xi<\alpha\right\}$ is not AW-convergent to the function $f$, which completes the proof.

Theorem 8.36 cannot be generalized for the class $\mathcal{F}(X, Y)$, which has been shown in Example 8.15. The considered there sequence of continuous functions is pointwise convergent and is not quasi-uniformly convergent, since the limit function is not continuous one.

Theorem 8.37. Let $X$ be a pseudo-compact space, $(Y, \rho)$ be a metric space, let $\left\{f_{j}: j \in J\right\}$ be a net of functions $f_{j}: X \longrightarrow Y$. The net $\left\{f_{j}: j \in J\right\}$ is $W$-convergent to $f: X \longrightarrow Y$ if and only if it is uniformly convergent to $f$.

Proof. Let $X$ be a pseudo-compact topological space. It is clear that if a net $\left\{f_{j}: j \in J\right\}$ is W-convergent to $f: X \longrightarrow Y$ then it is uniformly convergent.

Assume that $\left\{f_{j}: j \in J\right\}$ is uniformly convergent and take any $\varphi$ from $\mathcal{C}\left(X, \mathbb{R}^{+}\right)$. By pseudo-compactness of $X$, there exists a positive number $\delta$ such that $\varphi(x)>\delta$ for each $x \in X$, because otherwise $\frac{1}{\varphi}$ would be continuous and unbounded. Then there exists $j_{0} \in J$ such that

$$
\rho\left(f_{j}(x), f(x)\right)<\delta<\varphi(x)
$$

for each $x \in X$ and for each $j \in J$ such that $j \geq j_{0}$. It follows then that $\left\{f_{j}: j \in J\right\}$ is $\mathrm{W}$-convergent to $f$.

Theorem 8.38. Let $X$ be a completely regular topological space. If $X$ is not pseudo-compact then there is a sequence $\left\{f_{n}: n \in \mathbb{N}\right\}$, where $f_{n}: X \longrightarrow[0,1]$, which is uniformly convergent and it is not $W$-convergent.

Proof. Suppose that $X$ is not pseudo-compact. Then there exists a continuous and unbounded function $g: X \longrightarrow \mathbb{R}^{+}$. We can find a sequence $\left(x_{n}\right)_{n=1}^{\infty}$ of points from $X$ such that $\lim _{n \rightarrow \infty} g\left(x_{n}\right)=\infty$.

Let $\varphi=\frac{1}{|g|+1}$. Of course $\varphi \in \mathcal{C}\left(X, \mathbb{R}^{+}\right)$and $\lim _{n \rightarrow \infty} \varphi\left(x_{n}\right)=0$. Define $\left(f_{n}\right)_{n=1}^{\infty}$ letting $f_{n}(x)=\varphi\left(x_{n}\right)$ for each $x \in X$. It is obvious that $\left(f_{n}\right)_{n=1}^{\infty}$ is uniformly convergent to the zero function $f$. But 
110 Robert Drozdowski, Jacek Jędrzejewski, Stanisław Kowalczyk, Agata Sochaczewska

$$
\left|f_{n}\left(x_{n}\right)-f\left(x_{n}\right)\right|=\varphi\left(x_{n}\right)
$$

for each $n \in \mathbb{N}$ and hence $\left(f_{n}\right)_{n=1}^{\infty}$ is not W-convergent to function $f$.

Corollary 8.39. Let $X$ be a completely regular topological space. The following conditions are equivalent.

1. $X$ is pseudo-compact.

2. Uniform convergence and $W$-convergence of nets of functions are equivalent in $\mathcal{C}(X,[0,1])$.

3. Uniform convergence and $W$-convergence of nets of functions are equivalent in $\mathcal{F}(X,[0,1])$.

4. For each metric space $(Y, \rho)$ uniform convergence and $W$-convergence of nets of functions are equivalent in $\mathcal{C}(X, Y)$.

5. For each metric space $(Y, \rho)$ uniform convergence and $W$-convergence of nets of functions are equivalent in $\mathcal{F}(X, Y)$.

Remark 8.40. In [10] there are shown deeper connections between uniform convergence and Whitney convergence.

Theorem 8.41. For a compact topological space $X$ and metric space $(Y, \rho)$ pointwise convergence and quasi-uniform convergence of nets of functions are equivalent in $\mathcal{C}(X, Y)$.

Proof. Let $(Y, \rho)$ be a metric space. It is clear that if a net $\left\{f_{j}: j \in J\right\}$ from $\mathcal{C}(X, Y)$ is quasi-uniformly convergent to some $f: X \longrightarrow Y$ then it is pointwise convergent.

Let a net $\left\{f_{j}: j \in J\right\}$ from $\mathcal{C}(X, Y)$ be pointwise convergent to some continuous function $f: X \longrightarrow Y$. By Theorem 8.21, $\left\{f_{j}: j \in J\right\}$ is quasi-uniformly convergent to $f$.

Example 8.17 shows that pointwise convergence and quasi-uniform convergence of sequences of functions are not equivalent in $\mathcal{F}(X, Y)$, even if $X=Y=[0,1]$.

Similarly, Example 8.18 establishes that almost uniform convergence of sequences does not imply uniform convergence of sequences even in the space $\mathcal{C}([0,1],[0,1])$. This example shows simultaneously that almost Whitney convergence of sequences does not imply uniform convergence of sequences in $\mathcal{C}([0,1],[0,1])$. 


\title{
References
}

[1] C. Arzelá, Sulle serie di funzioni, Mem. della R. Accad. delle Sci. dell'Inst. di Bologna, ser. 5(8) (1899-1900), 130-186, 701-744.

[2] G. Di Maio, L. Hola, R. A. McCoy, Topologies on the space of continuous functions, Topology Appl. 86 (1998), 105-122.

[3] R. Drozdowski, J. Jędrzejewski, A. Sochaczewska, On the quasi-uniform convergence, Scientific Issues, Jan Długosz University in Częstochowa, Mathematics, XVI (2011), 19-22.

[4] J. Ewert, On the quasi-uniform convergence of transfinite sequences of functions, Acta Math. Univ. Comenianae, LXII (1993), 221-227.

[5] J. Ewert, Almost uniform convergence, Period. Math. Hungar. 26(1) (1993), 77-84.

[6] J. Ewert, J. Jędrzejewski, Between Arzelá and Whitney convergence, Real Anal. Exchange 29(1) (2004), 257-264.

[7] R. Ger, J. Jędrzejewski, Riemann integrability and quasi-uniform convergence, Jan Długosz University in Częstochowa, Scientific Issues, Mathematics XV, Częstochowa (2010), 31-34.

[8] L. Holà, R. A. McCoy, Compactness in the fine and related topologies, Topology Appl. 109 (2001), 183-190.

[9] J. Jędrzejewski, Almost uniform convergence of Riemann integrable functions, in Real functions, Density Topology and Related Topics, Dedicated to Professor Władysław Wilczyński, Wydawnictwo Uniwersytetu Łódzkiego 2011, 117-121.

[10] S. Kowalczyk On Whitney convergence, J. Appl. Anal. 15(1) (2009), 139-148.

[11] S. Kowalczyk, On local Whitney convergence, Scientific Issues, Jan Długosz University in Częstochowa, Mathematics XVII (2012), 57-68.

[12] M. Predoi, Sur la convergence quasi-uniforme, Period. Math. Hungar. 10(1) (1979), 31-49.

[13] B. Szökefalvi-Nagy, Theory of Real Functions and Orthogonal Expansions, Akademiai Kiado, Budapest 1964.

[14] H. Whitney, Differentiable manifolds, Ann. of Math. 37(3) (1936), 645-680.

\author{
ROBERT DROZDOWSKI \\ Institute of Mathematics, Pomeranian Academy \\ ul. Arciszewskiego 22d, 76-200 Słupsk, Poland \\ E-mail: r.drozdowski@wp.pl
}

\section{JACEK JĘDRZEJEWSKI}

Institute of Mathematics and Computer Science, Jan Długosz University in Częstochowa al. Armii Krajowej 13/15, 42-200 Częstochowa, Poland

E-mail: jacek.m.jedrzejewski@gmail.com 
112 Robert Drozdowski, Jacek Jędrzejewski, Stanisław Kowalczyk, Agata Sochaczewska

STANISEW KOWALCZYK

Institute of Mathematics, Pomeranian Academy ul. Arciszewskiego 22d, 76-200 Słupsk, Poland

E-mail: stkowalcz@onet.eu

\section{Agata SochaczewsKa}

Institute of Mathematics, Pomeranian Academy ul. Arciszewskiego 22d, 76-200 Słupsk, Poland

E-mail: sochaczedapsl.edu.pl 